\title{
Vision-Based Nonlinear Feedback Control of a Ball on Ball System With a Programmable Logic Controller
}

\author{
Matthias Bibl $^{* a)}$ Member, Christoph Zech ${ }^{* *}$ Non-member \\ Georg Schitter* Non-member
}

(Manuscript received May 1, 2018, revised Feb. 1, 2019)

\begin{abstract}
This paper shows how to implement a vision-based control for a Ball on Ball system. The control task is to stabilize a ball on top of another ball in the unstable upper rest position. In order to stabilize the upper ball, the position of the upper ball has to be measured. This is done optionally by using two triangulation sensors or an industrial camera. To stabilize the upper ball in the unstable upper rest position, a PD controller, a linear quadratic regulator, and a flatness-based nonlinear controller are designed. By using these controllers and the designed image processing, which are implemented entirely on a standard industrial programmable logic controller, it is possible to stabilize the upper ball with both measurement types. The disturbance rejection of the system is shown, and the resulting RMS position error when stabilizing the upper ball is $1.8 \mathrm{mrad}$.
\end{abstract}

Keywords: ball on ball, nonlinear control, vision based measurement, programmable logic controller

\section{Introduction}

Vision based measurement is getting increasingly important in automation systems. Examples are object recognition to grab objects with a robot ${ }^{(1)}$ or obstacle recognition for path planning of autonomous moving systems ${ }^{(2)}$. As the computing power of modern processors is continuously increasing, it is possible to use vision based methods for position detection.

To evaluate the capabilities of image processing on modern programmable logic controllers (PLCs) the task of balancing a ball on top of a second ball is chosen. The measuremnt of the ball should be done by an standard industrial camera.

Ball on ball control has been reported in literature by using two bowling balls of the same size stabilizing the upper ball ${ }^{(3)}$, with position measurement done by two triangulation sensors. Another ball on ball setup has been reported $^{(4)(5)}$, where the lower ball is 9 times bigger than the upper ball. In this setup the position measurement is done with a $128 \times 101$ pixel CMOS sensor. The image processing to determine the upper ball position is done on a FPGA.

The contribution of this paper is the demonstration of feasibility of performing control and image processing for a ball on ball setup on a standard industrial PLC. Due to the implementation of direct access to the camera in the used PLC system, the image processing can be done entirely on the PLC.

In Sec. 2 the modeling of the mechanical and the electrical system is done. The controllers to stabilize the system

a) Correspondence to: Matthias Bibl. E-mail: matthias.bibl@ tuwien.ac.at

* Automation and Control Institute, Vienna University of Technology

Gußhausstraße 27-29, A-1040 Vienna, Austria

** Beckhoff Automation GmbH

Schloss 2B/Top 3, A-2542 Kottingbrunn, Austria are designed in Sec. 3. Sec. 4 shows the experimental setup and parameters. The results are shown in Sec. 5 and are concluded in Sec. 6.

\section{Modeling of the System}

2.1 Mechanical System In Fig. 1 the schematic structure of the system is shown. The coordinate systems are labeled $c f_{i}$, where $i$ denotes the number of the coordinate system in the drawing. To derive the equations of motion the Euler-Lagrange equation is used. The dynamics of the balls are calculated in their respective body coordinate systems $c f_{i}, i \in[1,2]$ and are then transformed into the same coordinate system. To describe the rotation of both balls Euler angles are used and the order of rotations is chosen to the $\mathrm{z}^{\prime} \mathrm{Xz}$-convention ${ }^{(6)}$. With the Euler angles of the $i$-th ball $\left(\alpha_{i}, \beta_{i}, \gamma_{i}\right)$ the full rotation matrix can be found as

$$
\mathbf{R}_{z^{\prime} x z}=\mathbf{R}_{z^{\prime}}\left(\alpha_{i}\right) \cdot \mathbf{R}_{x}\left(\beta_{i}\right) \cdot \mathbf{R}_{z}\left(\gamma_{i}\right)=
$$

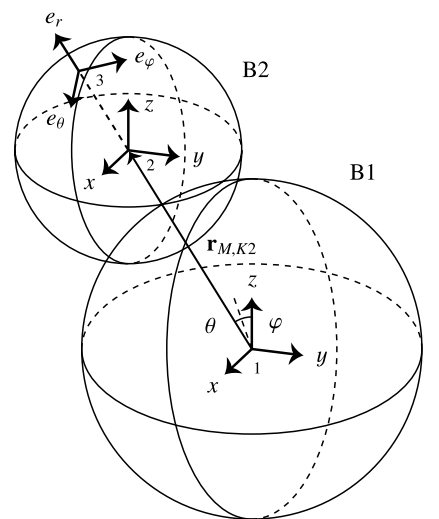

Fig. 1. Schematic of the lower (B1) and the upper (B2) ball with the coordinate systems and the model variables 


$$
\left(\begin{array}{ccc}
c_{\alpha, i} c_{\gamma, i}-s_{\alpha, i} c_{\beta, i} s_{\gamma, i} & s_{\alpha, i} c_{\gamma, i}+c_{\alpha, i} c_{\beta, i} s_{\gamma, i} & s_{\beta, i} s_{\gamma, i} \\
-c_{\alpha, i} s_{\gamma, i}-s_{\alpha, i} c_{\beta, i} c_{\gamma, i} & -s_{\alpha, i} s_{\gamma, i}+c_{\alpha, i} c_{\beta, i} c_{\gamma, i} & s_{\beta, i} c_{\gamma, i} \\
s_{\alpha, i} s_{\beta, i} & -c_{\alpha, i} s_{\beta, i} & c_{\beta, i}
\end{array}\right)
$$

with the cosine $c_{x, i}$ and the sine $s_{x, i}$ of the angle $x_{i}$. The kinetic energy of both balls, which is needed for the EulerLagrange equation, can be found by the skew-symmetric ma$\operatorname{trix} \mathbf{S}^{(7)}$ and is given by

$$
\mathbf{S}=\dot{\mathbf{R}} \mathbf{R}^{T}=\left(\begin{array}{ccc}
0 & -\omega_{z} & \omega_{y} \\
\omega_{z} & 0 & -\omega_{x} \\
-\omega_{y} & \omega_{x} & 0
\end{array}\right)
$$

So the angular velocities can be determined to

$$
\begin{aligned}
\omega_{x} & =\sin (\alpha) \sin (\beta) \dot{\gamma}+\cos (\alpha) \dot{\beta} \ldots \\
\omega_{y} & =-\cos (\alpha) \sin (\beta) \dot{\gamma}+\sin (\alpha) \dot{\beta} \\
\omega_{z} & =\dot{\alpha}+\cos (\beta) \dot{\gamma} . \cdots \ldots \ldots \ldots
\end{aligned}
$$

By using a transformation to the coordinate system $c f_{3}$ the coordinate systems can be brought to the same coordinate system. When assuming that each of the two balls is axially symmetric the moment of inertia for each ball is given by

$$
I_{i}=\frac{2}{5} m_{i} r_{i}^{2}
$$

where $m_{i}$ denotes the mass of the ball and $r_{i}$ the radius of the ball. The potential and kinetic energy of the balls can be found, where the potential energy of the lower ball is zero because it can only make rotational but no translational movements.

Two boundary conditions for the system can be determined. The first one is that the upper ball is always on top of the lower ball, so the distance between the centers of the two balls is always the sum of the radii of the two balls. So the first condition is

$$
\left|\mathbf{r}_{M, K 2}\right|=\sqrt{x^{2}+y^{2}+z^{2}}=\sqrt{\left(r_{1}+r_{2}\right)^{2}}=R . \cdots \cdots
$$

The second boundary condition is, that assuming there is no slip between the balls, the rolling condition

$$
\mathbf{r}_{2} \times \omega_{2}+\mathbf{v}=\mathbf{r}_{1} \times \omega_{1}
$$

with the vector of the angular velocities $\omega_{i}$ of the balls and the translational velocity $\mathbf{v}$ of the upper ball, has to be fulfilled. By choosing an appropriate material for the balls and limiting the motor moments it can be ensured, that no slip occurs between the two balls around the upper rest position.

The generalized coordinates are chosen to be

$$
\mathbf{q}=\left[\begin{array}{llllllll}
\alpha_{1} & \beta_{1} & \gamma_{1} & \varphi & \theta & \alpha_{2} & \beta_{2} & \gamma_{2}
\end{array}\right]^{T},
$$

with the Euler angles $\alpha_{i}, \beta_{i}$ and $\gamma_{i}$ of each ball, the generalized velocities are

$$
\dot{\mathbf{q}}=\left[\begin{array}{llllllll}
\omega_{1, \varphi} & \omega_{1, \theta} & \omega_{1, r} & \omega_{\varphi} & \omega_{\theta} & \omega_{2, \varphi} & \omega_{2, \theta} & \omega_{2, r}
\end{array}\right]^{T},
$$

and the generalized forces are

$$
\tau=\left[\begin{array}{llllllll}
M_{1} & M_{2} & 0 & 0 & 0 & 0 & 0 & 0
\end{array}\right]^{T} .
$$

By using the Euler-Lagrange equation

$$
\frac{d}{d t}\left(\frac{d L}{d \dot{q}_{j}}\right)-\frac{d L}{d q_{j}}=\tau
$$

with the Lagrangian

$$
L=T_{K 1}+T_{K 2}-V_{K 1}-V_{K 2}, \cdots
$$

where $T$ denotes the kinetic energy of the balls and $V$ the potential energy and by using the Euler-Lagrange equation of the first kind the term

$$
\mathbf{A}(\mathbf{q}) \lambda=\sum_{i} \frac{\partial f_{i}}{\partial \dot{q}_{j}} \lambda_{i}, i \in k
$$

can be found and added to Eq. (10) to consider the $k$ boundary conditions of Eqs. (5) and (6). The resulting system can be written as

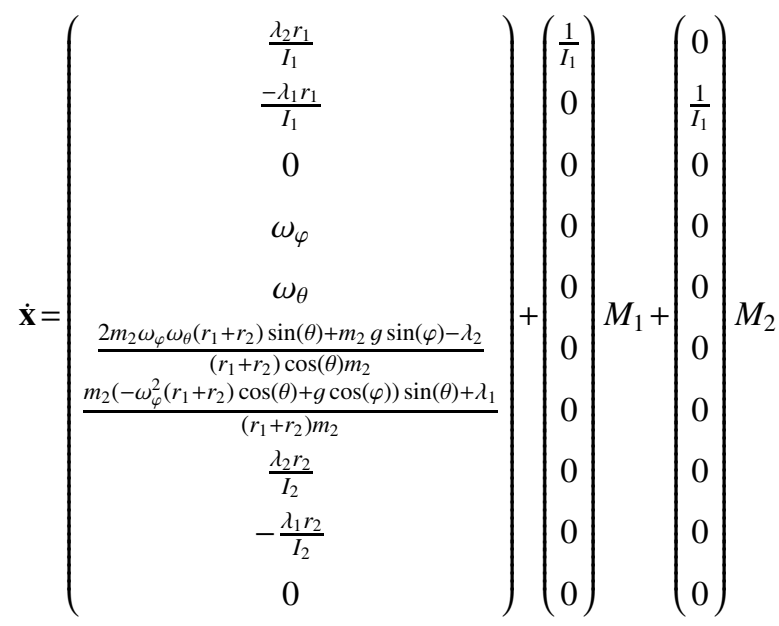

with the boundary conditions

$$
\begin{aligned}
& 0=r_{1} \omega_{1, \theta}-\left(r_{1}+r_{2}\right) \omega_{\theta}+r_{2} \omega_{2, \theta} \cdots \cdots \\
& 0=-r_{1} \omega_{1, \varphi}+\cos (\theta)\left(r_{1}+r_{2}\right) \omega_{\varphi}-r_{2} \omega_{2, \varphi}
\end{aligned}
$$

and the state vector

$$
\mathbf{x}=\left[\begin{array}{llllllllll}
\omega_{1, \varphi} & \omega_{1, \theta} & \omega_{1, r} & \varphi & \theta & \omega_{\varphi} & \omega_{\theta} & \omega_{2, \varphi} & \omega_{2, \theta} & \omega_{2, r}
\end{array}\right]^{T} .
$$

As the angular velocities $\omega_{2, \varphi}$ and $\omega_{2, \theta}$ can not be measured and are not needed for the control task their dynamic equations are solved for the lagrange multipliers. By differentiating with respect to time the terms can be substituted in Eq. (13) and the final form can be found. Because of the fairly large result the final result is not shown.

2.2 Electrical System The lower ball is driven by three permanent magnet synchronous motors (PSM). The model of the PSM is ${ }^{\left({ }^{8}\right)}$

$$
\begin{aligned}
& \dot{\mathbf{x}}=\left(\begin{array}{c}
\frac{1}{l_{s}}\left(-i_{s d} r_{s}+\omega_{m} l_{s} i_{s q}\right) \\
\frac{1}{l_{s}}\left(-i_{s q} r_{s}-\omega_{m}\left(l_{s} i_{s d}+\Psi_{M}\right)\right) \\
\frac{1}{\tau_{m}}\left(i_{s q} \Psi_{M}+\tau_{L}\right)
\end{array}\right)+\left(\begin{array}{c}
\frac{1}{l_{s}} \\
\frac{1}{l_{s}} \\
0
\end{array}\right)\left[\begin{array}{c}
u_{s d} \\
u_{s q} \\
0
\end{array}\right] \\
& y=i_{s q} \Psi_{M}
\end{aligned}
$$

where $l_{s}$ denotes the stator inductance, $r_{s}$ is the stator resistance $\Psi_{M}$ is the interlinking magnetic flux and $\tau_{L}$ is the load 
moment. The state vector $\mathbf{x}=\left[\begin{array}{lll}i_{s d} & i_{s q} & \omega_{m}\end{array}\right]$ denotes the stator current $i$ in $d$ and $q$ direction and the rotational speed $\omega_{m}$. The input of the system is the stator voltage $u$ in $d$ and $q$ direction and the output is the mechanical torque $y=\tau_{M}$.

It can be assumed that the mechanical system is much slower than the electrical electrical system. When using the singular perturbation method ${ }^{(9)}$ by choosing the disturbance parameter $\epsilon=l_{s}=0$ the model for the PSM can be reduced to the algebraic equations

$$
\begin{aligned}
i_{s d} & =\frac{u_{s d}}{r_{s}}, \ldots \ldots \ldots \ldots \ldots \\
i_{s q} & =\frac{u_{s q}-\Psi_{M} \omega_{m}}{r_{s}} \text { and } . \\
\tau_{m} & =\frac{\Psi_{M} u_{s q}-\Psi_{M}^{2} \omega_{m}}{r_{s}} . \cdots
\end{aligned}
$$

When adding the time constant of the rotor inertia as a second disturbance parameter Eq. (19) can be reduced to

$$
\tau_{m}=-\tau_{L}
$$

This means that the electric subsystem is a through connection because it is much faster than the mechanical one.

2.3 Input Transformation In Eq. (13) the system inputs are the torques $M_{1}$ and $M_{2}$. In this publication the angular velocities of the lower ball are controlled and not the torques, such that an input transformation has to be found. By solving the system model of Sec. 2.1 for the torques $M_{1}$ and $M_{2}$ and substituting the remaining equations the new system with the inputs $\omega_{1, \varphi}$ and $\omega_{1, \theta}$ is found. Since the two states $\omega_{1, r}$ and $\omega_{2, r}$ do not influence any other states and can not be actuated or measured they are neglected. When all higher terms of $\omega_{\varphi}$ and $\omega_{\theta}$ are neglected the system can be reduced to

$$
\mathbf{f}(\mathbf{x}, \mathbf{u})=\left(\begin{array}{c}
\omega_{\varphi}+\frac{I_{2} r_{1}}{\left(r_{1}+r_{2}\right)\left(m_{2} r_{2}^{2}+I_{2}\right) \cos (\theta)} \omega_{1, \varphi} \\
\omega_{\theta}+\frac{I_{2} r_{1}}{\left(r_{1}+r_{2}\right)\left(m_{2} r_{2}^{2}+I_{2}\right)} \omega_{1, \theta} \\
\frac{g m_{2} r_{2}^{2} \sin (\varphi)}{\left(r_{1}+r_{2}\right)\left(m_{2} r_{2}^{2}+I_{2}\right) \cos (\theta)} \\
\frac{g \cos (\theta) m_{2} r_{2}^{2} \sin (\theta)}{\left(m_{2} r_{2}^{2}+I_{2}\right)\left(r_{1}+r_{2}\right)}
\end{array}\right)
$$

Assuming that the angles $\varphi$ and $\theta$ are small, the simplification $\cos (\theta) \approx 1$ can be used and the system

$$
\mathbf{f}(\mathbf{x}, u)=\underbrace{\left(\begin{array}{c}
\omega_{\varphi} \\
\frac{g m_{2} r_{2}^{2} \sin (\varphi)}{\left(r_{1}+r_{2}\right)\left(m_{2} r_{2}^{2}+I_{2}\right)}
\end{array}\right)}_{\mathbf{f}(\mathbf{x})}+\underbrace{\left(\begin{array}{c}
\left.\frac{I_{2} r_{1}}{\left(r_{1}+r_{2}\right)\left(m_{2} r_{2}^{2}+I_{2}\right)}\right) \\
0
\end{array}\right)}_{\mathbf{g}(\mathbf{x})} u, \cdots
$$

with the reduced state vector $\mathbf{x}=\left[\begin{array}{ll}\varphi & \omega_{\varphi}\end{array}\right]^{T}$ can be found. This system is valid for the $\varphi$ and the $\theta$ angle. So the system splits into two indentical decoupled systems around the upper rest position.

2.4 Linearisation For a first analysis the system of Eq. (21) is linearized around the upper rest position where $\varphi=\theta=0$ and $\dot{\mathbf{x}}_{u=0}=\mathbf{0}$. The system can be determined as

$$
\mathbf{A}=\left(\begin{array}{cccc}
0 & 0 & 1 & 0 \\
0 & 0 & 0 & 1 \\
a_{1} & 0 & 0 & 0 \\
0 & a_{1} & 0 & 0
\end{array}\right) \text {, with }
$$

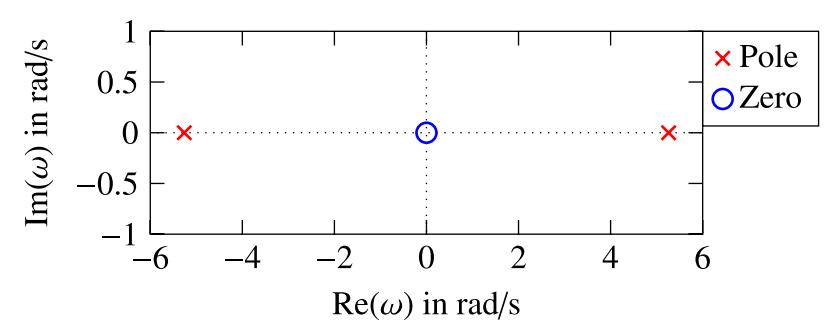

Fig. 2. Pole zero map of the linearized system of Eq. (25). Due to the pole in the right half plane the system is unstable

$$
a_{1}=\frac{g m_{2} r_{2}^{2}}{\left(r_{1}+r_{2}\right)\left(m_{2} r_{2}^{2}+I_{2}\right)}
$$

and the input matrix

$$
\begin{aligned}
& \mathbf{B}=\left(\begin{array}{cc}
b_{1} & 0 \\
0 & b_{1} \\
0 & 0 \\
0 & 0
\end{array}\right), \text { with } \ldots . . \\
& b_{1}=\frac{I_{2} r_{1}}{\left(m_{2} r_{2}^{2}+I_{2}\right)\left(r_{1}+r_{2}\right)} .
\end{aligned}
$$

It can easily be seen that also the linearized system splits into two identical systems of the form

$$
\begin{aligned}
\dot{\mathbf{x}} & =\left(\begin{array}{cc}
0 & 1 \\
a_{1} & 0
\end{array}\right) \\
\mathbf{y} & =\underbrace{\left(\begin{array}{cc}
1 & 0
\end{array}\right)}_{\mathbf{C}} \mathbf{x}+\underbrace{\left(\begin{array}{c}
b_{1} \\
0
\end{array}\right)}_{\mathbf{D}} u
\end{aligned}
$$

with the state vectors $\mathbf{x}_{1}=\left[\begin{array}{ll}\varphi & \omega_{\varphi}\end{array}\right]^{T}$ and $\mathbf{x}_{2}=\left[\begin{array}{ll}\theta & \omega_{\theta}\end{array}\right]^{T}$. The resulting system is much easier to analyze. It can be shown that each subsystem has a pole in the left half-plane and one in the right half-plane. A pole zero map of the linearized system is shown in Fig. 2.

The observability matrix $\mathscr{O}(\mathbf{A}, \mathbf{C})=\left[\begin{array}{ll}\mathbf{C} & \mathbf{C A}\end{array}\right]^{T}$ has full rank $n=2$, even if the angular velocity $\omega_{1, \varphi}$ can not be measured, therefore the system is fully observable. Also the controllability matrix $\mathscr{R}(\mathbf{A}, \mathbf{B})=\left[\begin{array}{ll}\mathbf{B} & \mathbf{A B}\end{array}\right]$ has full rank and therefore the system is fully controllable. Due to the decoupling of the two axes a controller can be designed for one axis and then also be used for the second axis.

\section{Control Design}

Based on the derived mathematical model a controller is designed to stabilize the upper ball in the upper rest position. In Table 1 the used parameters for the system are given. The control layout can be seen in Fig. 3, where $\varphi_{d}$ denotes the desired angle of the upper ball, $\omega_{1, d}$ is the desired velocity of the lower ball, $I_{s}$ is the current, and $U_{s}$ the voltage of the PSM.

First a PD controller is designed in Sec. 3.1. To improve the system performance a linear quadratic regulator is designed for the system in Sec. 3.2. Due to the flatness of the system and to further improve the performance of the system a nonlinear flatness based controller is designed in Sec. 3.3.

3.1 PD Controller Design Using the parameters of Table 1 the system dynamics of Eq. (25) leads to

$$
\dot{\mathbf{x}}_{1}=\left(\begin{array}{cc}
0 & 1 \\
28.03 & 0
\end{array}\right) \mathbf{x}_{1}+\left(\begin{array}{c}
0.1714 \\
0
\end{array}\right) u
$$


Table 1. Model parameters used for the simulation

\begin{tabular}{l|l} 
Parameter & Value \\
\hline$r_{1}$ & $150 \mathrm{~mm}$ \\
$r_{2}$ & $100 \mathrm{~mm}$ \\
$m_{1}$ & $5 \mathrm{~kg}$ \\
$m_{2}$ & $3 \mathrm{~kg}$ \\
$M_{\max }$ & $5 \mathrm{Nm}$
\end{tabular}

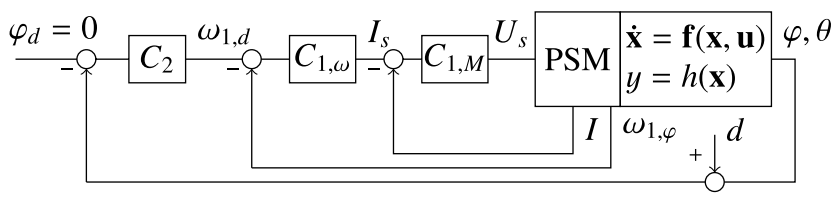

Fig. 3. Used control structure to stabilize the system. The two inner loops control the PSM current and angular velocity, the outer loop controls the angle of the upper ball

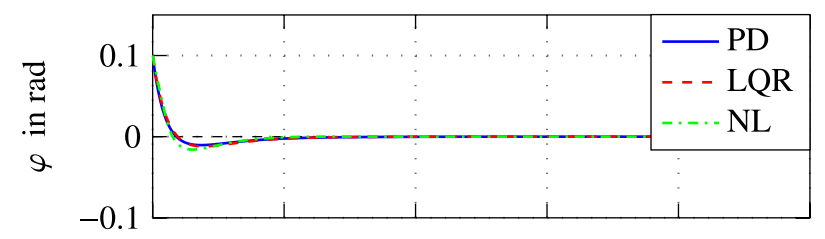

(a)

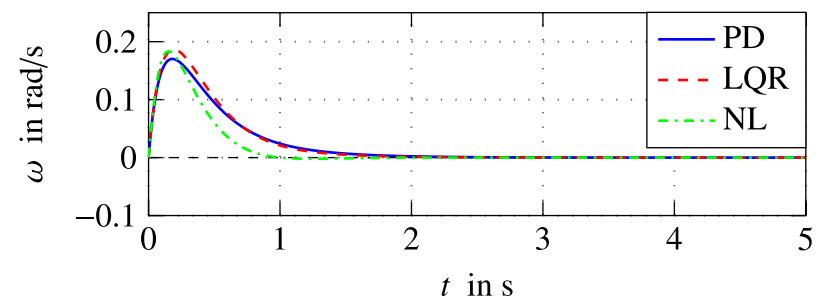

(b)

Fig. 4. Angle $\varphi$ of the upper ball over time for a initial deflection of $\varphi_{0}=0.1 \mathrm{rad}$ (a) and the corresponding angular velocity $\omega_{\varphi}$ (b) for the PD controller, the linear quadratic regulator and the nonlinear controller

$$
\mathbf{y}_{1}=\left(\begin{array}{ll}
1 & 0
\end{array}\right) \mathbf{x}_{1}
$$

This system is used for the linear control design.

Because of the integrating behavior of the system a PD controller in generalized system coordinates of the form

$$
\tau=\mathbf{K}_{\mathbf{p}}\left(\mathbf{q}_{d}-\mathbf{q}\right)-\mathbf{K}_{\mathbf{d}} \dot{\mathbf{q}}+\mathbf{g}(\mathbf{q}), \cdots
$$

with $\mathbf{K}_{\mathbf{p}}$ beeing the proportional gain matrix, $\mathbf{K}_{\mathbf{D}}$ the differential gain matrix, $\mathbf{g}(\mathbf{q})$ the compensation term for potential forces, and the desired coordinates $\mathbf{q}_{d}$, is sufficient to stabilize the unstable upper rest position. To design the PD control the tuning rule presented in ${ }^{(10)}$ is used. By choosing a crossover frequency of $\omega_{C}=35 \mathrm{~s}^{-1}$ and empirical optimization of the gain $K_{d}=11$, the system can be stabilized. The response of the system is shown in Fig. 4 for a initial deflection of $0.1 \mathrm{rad}$.

3.2 LQR Controller Design A second method to stabilize the upper rest position is the linear quadratic regulator $(\mathrm{LQR})^{(11)}$

$$
u_{k}=-\mathbf{K} \mathbf{x}_{k}
$$

The LQR is designed directly for the time discrete system and therefore a sampling time has to be chosen, such that the sampling frequency is 5 to 25 times higher than the system bandwidth $^{(11)(12)}$. This leads to a sampling time between $86 \mathrm{~ms}$ and $17 \mathrm{~ms}$. As the PLC can easily achieve a sampling time of $10 \mathrm{~ms}$ for the implementation, a sampling time of $10 \mathrm{~ms}$ is chosen. The discretised system is given by

$$
\begin{gathered}
\mathbf{x}_{\mathbf{1}, \mathbf{k}+\mathbf{1}}=\underbrace{\left(\begin{array}{cc}
1.001 & 0 \\
0.2804 & 1.001
\end{array}\right)}_{\boldsymbol{\Phi}} \mathbf{x}_{\mathbf{1}, \mathbf{k}}+\underbrace{\left(\begin{array}{c}
0.001715 \\
0.0002405
\end{array}\right)}_{\Gamma} u_{k} \\
\mathbf{y}_{\mathbf{1}, \mathbf{k}}=\underbrace{\left(\begin{array}{ll}
1 & 0
\end{array}\right)}_{\mathbf{C}} \mathbf{x}_{\mathbf{1}, \mathbf{k}} \cdot \ldots \ldots \ldots \ldots \ldots \ldots \ldots \ldots \ldots \ldots \ldots \ldots \ldots \ldots \ldots
\end{gathered}
$$

By using the cost function

$$
J=\sum_{k=0}^{N-1}\left(\mathbf{x}_{\mathbf{k}}^{\mathbf{T}} \mathbf{Q} \mathbf{x}_{\mathbf{k}}+\mathbf{u}_{\mathbf{k}}^{\mathbf{T}} \mathbf{R} \mathbf{u}_{\mathbf{k}}+\mathbf{2} \mathbf{x}_{\mathbf{k}}^{\mathbf{T}} \mathbf{N} \mathbf{u}_{\mathbf{k}}\right)+\mathbf{x}_{\mathbf{N}}^{\mathbf{T}} \mathbf{S} \mathbf{x}_{\mathbf{N}}
$$

with the weighting matrices $\mathbf{Q}, \mathbf{R}, \mathbf{N}$ and $\mathbf{S}$ with respect to $\mathbf{u}_{N-1}$ and the weighting matrices

$$
\mathbf{Q}=\left(\begin{array}{cc}
7000 & 0 \\
0 & 1
\end{array}\right), \quad R=10 \text { and } \mathbf{N}=\mathbf{0} \ldots \ldots \ldots \ldots
$$

the feedback gain vector

$$
\mathbf{k}=\left(\begin{array}{ll}
65.0452 & 11.3482
\end{array}\right)
$$

can be found. This feedback vector is similar to the previously designed PD controller ${ }^{(10)}$. In Fig. 4 the response of the system to an initial deflection of $0.1 \mathrm{rad}$ is shown.

\subsection{Flatness Based Nonlinear Controller Design}

A third control strategy is to design a nonlinear controller for the system of Eq. (22). As discussed earlier the coupling between the orthogonal axes is weak and therefore the system can be seen as two orthogonal ball on wheel systems. It can be shown that a ball on wheel system with the torque as input is flat ${ }^{(13)}$. A system that can be linearized by feedback must have the form ${ }^{(9)}$

$$
\dot{\mathbf{x}}=\mathbf{A x}+\mathbf{B} \gamma(\mathbf{x})(u-\alpha(\mathbf{x}))
$$

which is the case for Eq. (22). To verify that a flat output exists the matrix

$$
\mathbf{G}=\left[\begin{array}{ll}
\mathbf{g} & \mathbf{L}_{\mathbf{f}} \mathbf{g}(\mathbf{x})
\end{array}\right]=\left(\begin{array}{cc}
\frac{I_{2} r_{1}}{\left(r_{1}+r_{2}\right)\left(m_{2} r_{2}^{2}+I_{2}\right)} & 0 \\
0 & -\frac{g m_{2} r_{2}^{2} I_{2} r_{1} \cos (\varphi)}{\left(r_{1}+r_{2}\right)^{2}\left(m_{2} r_{2}^{2}+I_{2}\right)^{2}}
\end{array}\right)
$$

with $\mathbf{f}$ and $\mathbf{g}$ from Eq. (22) must have full rank. As this is true it remains to show that the distribution $D=\operatorname{span}\{\mathbf{g}\}$ is involute in an area around $\overline{\mathbf{x}}$. Because $D$ has full rank the distribution is a basis of the complete $\mathbb{R}$, and therefore the Lie-brackets of every combination $\mathbf{f}_{\mathbf{i}}(\mathbf{x}), \mathbf{f}_{\mathbf{j}}(\mathbf{x}) \in D$ of vector fields has to fulfill $\left[\mathbf{f}_{\mathbf{i}}(\mathbf{x}), \mathbf{f}_{\mathbf{j}}(\mathbf{x})\right] \in D$. Therefore it is shown that the system must have an output with relative order $r=n$. A possible flat output is simply found by

$$
y=h(\mathbf{x})=\omega_{\varphi}
$$

By differentiating the output it can be shown that $\dot{y}$ is independent of the input $\omega_{1, \varphi}$, but $\ddot{y}$ is function of $\omega_{1, \varphi}$. Because 
the input occurs at the second derivative the relative order $r$ is 2 , which is also the number of system states, such that the output is flat. This means a feedback law according to the form of ${ }^{(9)}$

$$
u=\frac{1}{\mathbf{L}_{\mathbf{g}} h(x)}\left(-\mathbf{L}_{\mathbf{f}} h(x)+v\right)
$$

can be derived. To set a desired characteristic polynomial of the linearized system the new input $v$ is chosen to be

$$
v=-\sum_{j=1}^{n} a_{j-1} \mathbf{L}_{\mathbf{f}}^{j-1} h(\mathbf{x})
$$

This results in a denominator of the form $s r+a r-1 s r-1$ $+\ldots+a 0$ and the complete feedback law is given by

$$
\begin{aligned}
u= & \frac{1}{\mathbf{L}_{\mathbf{g}} \mathbf{L}_{\mathbf{f}} h(\mathbf{x})}\left(-\mathbf{L}_{\mathbf{f}}^{2} h(\mathbf{x})+v\right)=\ldots \ldots \ldots \ldots \ldots(38) \\
& -\frac{7}{2} \frac{\left(r_{1}+r_{2}\right)\left[m_{2} g \cos (\varphi) r_{2}^{2} \omega_{\varphi}+a_{1} m_{2} g \sin (\varphi) r_{2}^{2}+\right.}{m_{2} g r_{2}^{2} r_{1} \cos (\varphi)} \\
& \underline{\left.\left(r_{1}+r_{2}\right)\left(m_{2} r_{2}^{2}+I_{2}\right)\left(a_{0} \omega_{\varphi}-v\right)\right]}
\end{aligned}
$$

with the coefficients $a_{j}$ of the desired characteristic polynomial. The coefficients are chosen to $(12,36)$ and the resulting poles of the transfer function are $(-6,-6)$, because these parameters yield good results in the simulation. The system response for a initial deflection of $0.1 \mathrm{rad}$ can be seen in Fig. 4. It can be seen that the behavior of the system for the angle $\varphi$ is for all three controllers nearly identical. The angular velocity $\omega_{\varphi}$ is controlled back to zero by the nonlinear controller in $\approx 1 \mathrm{~s}$ and by the other two controllers in $\approx 2 \mathrm{~s}$. Therefore it can be concluded that the nonlinear controller achieves the highest disturbance rejection. As the goal of the system is to stabilize the upper ball in the unstable upper rest position the disturbance rejection is the most important attribute.

The three designed controllers are designed for the simplified system models. To quantify the error by these simplifications the simulations are done for the reduced and the full nonlinear model. The results can be seen in Fig. 5. It turns out that the behavior for the angle is nearly identical and for the angular velocities a small deviation at the peak velocity can be seen. Therefore it is concluded that the controllers designed for the reduced system also work for the full nonlinear system.

3.4 State Observer It was assumed that all system states can be measured. This is true for the angles $\varphi$ and $\theta$ but not for the angular velocities $\omega_{\varphi}$ and $\omega_{\theta}$. To determine the velocities the angles can be differentiated with respect to time. This method is known to amplify measurement noise and may not provide good results. A different method is to use a state observer in form of a Kalman filter ${ }^{(14)}$. For the linear Kalman filter the matrices $\mathbf{Q}, \mathbf{R}$ and $\mathbf{N}$ need to be chosen, as well as the initial state of $\hat{\mathbf{x}}_{0}$. The filter is designed for the time-discrete system from Eq. (29). Furthermore the system is extended by the disturbance $\mathbf{w}_{k}$ and the sensor noise $\mathbf{v}_{k}$, and the matrices $\mathbf{G}$ and $\mathbf{H}$ are chosen to

$$
\mathbf{G}=\left(\begin{array}{c}
0.25 \\
1
\end{array}\right) \text { and } H=0 \ldots \ldots \ldots \ldots \ldots \ldots \ldots
$$

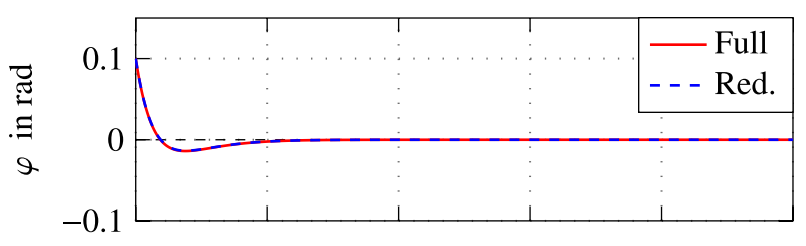

(a)

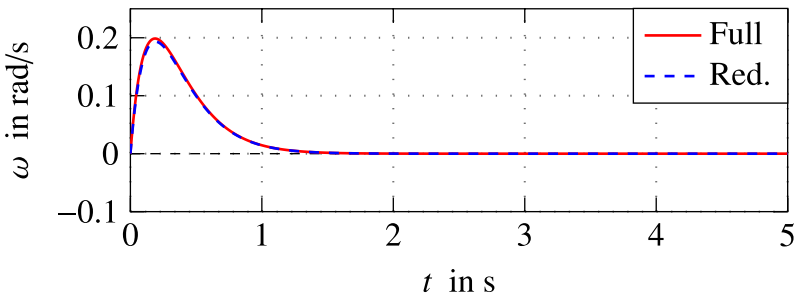

(b)

Fig. 5. Angle $\varphi$ of the upper ball for a initial deflection of $\varphi_{0}=0.1 \mathrm{rad}$ (a) and the corresponding angular velocity $\omega_{\varphi}$ (b) for the full nonlinear model and the reduced model

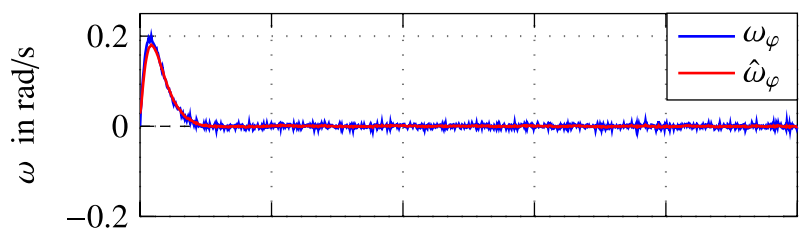

(a)

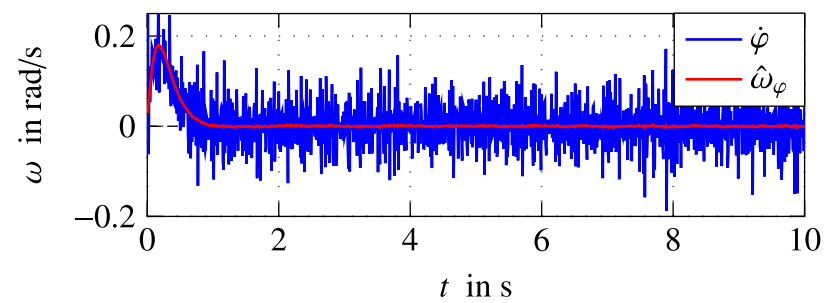

(b)

Fig. 6. The actual state $\omega_{\varphi}$ compared to the estimated state $\hat{\omega}_{\varphi}$ in the simulation (a) and the derivative of the angle $\varphi$ with respect to time compared to the estimated state $\hat{\omega}_{\varphi}$ for the real system (b)

so the disturbance mainly influences the not measured state $\omega_{\varphi}$. The disturbance $\mathbf{w}_{k}$ and the sensor noise $\mathbf{v}_{k}$ are assumed as one dimensional properties and their covariance matrices $\mathbf{Q}$ and $\mathbf{R}$ are therefore scalars. They are tuned empirically to

$$
Q=500000 \text { and } R=7 .
$$

This leads to the estimation matrix

$$
L=\left(\begin{array}{l}
0.5249 \\
2.2480
\end{array}\right)
$$

With these settings the Kalman filter achieves a good estimation of the state vector, even if the measurement noise is large. A comparison between the state $\omega_{\varphi}$ and the estimated state $\hat{\omega}_{\varphi}$ for the simulation can be seen in Fig. 6(a). For the real system a comparison between the derivative of the angle $\varphi$ with respect to time compared to the estimated state $\hat{\omega}_{\varphi}$ can be seen in Fig. 6(b). It can be seen, that the estimated state $\hat{\omega}_{\varphi}$ is much less noisier than the derivative of the angle $\varphi$ with respect to time. 


\section{Experimental Setup}

After modeling the system and designing the controllers a experimental setup is built. The parameters of the setup are shown in Table 2. A picture of the final setup can be seen in Fig. 7. The setup is driven by three Beckhoff PSM servo Motors AM8121 with a max. torque of $0.5 \mathrm{Nm}$. To increase the torque a Beckhoff AG2250 gearbox with a gear ratio of $1: 3$ is used. The gearbox is via a flexible jaw coupling connected with an omniwheel to drive the lower ball. A Beckhoff EL7211-9014 inverter is used to control the current and the angular velocity of each motor. TwinCAT from Beckhoff is used as the PLC software and the used industrial PC is a Beckhoff CX2030. The controllers are implemented in Matlab Simulink and then exported as a $\mathrm{C}++$ object. These objects can be directly imported and used in TwinCAT.

4.1 Triangulation Measurement (M1 \& M2) To determine the position of the upper ball two triangulation sensors IL 300 from Keyence are used. The two triangulation sensors measure the distance to the upper ball from which the angle $\varphi$ and $\theta$ can be calculated. In principle the task of calculating the two angles can be reduced to the task of finding the intersection of three spheres and is known as trilateration ${ }^{(15)}$. The sampling time for each sensor is $1 \mathrm{~ms}$.

4.2 Camera Based Measurement (M3) To detect the upper ball position also an industrial gigabit ethernet camera Manta G046C is used. It is mounted above the upper ball, so that the full ball and its movement can be observed. The camera uses a Sony ICX415 sensor with a

Table 2. Parameters of the lower and the upper ball

\begin{tabular}{c|c} 
Parameter & Value \\
\hline$m_{1}$ & $1.40 \mathrm{~kg}$ \\
$m_{2}$ & $3.56 \mathrm{~kg}$ \\
$r_{1}$ & $151 \mathrm{~mm}$ \\
$r_{2}$ & $101 \mathrm{~mm}$
\end{tabular}

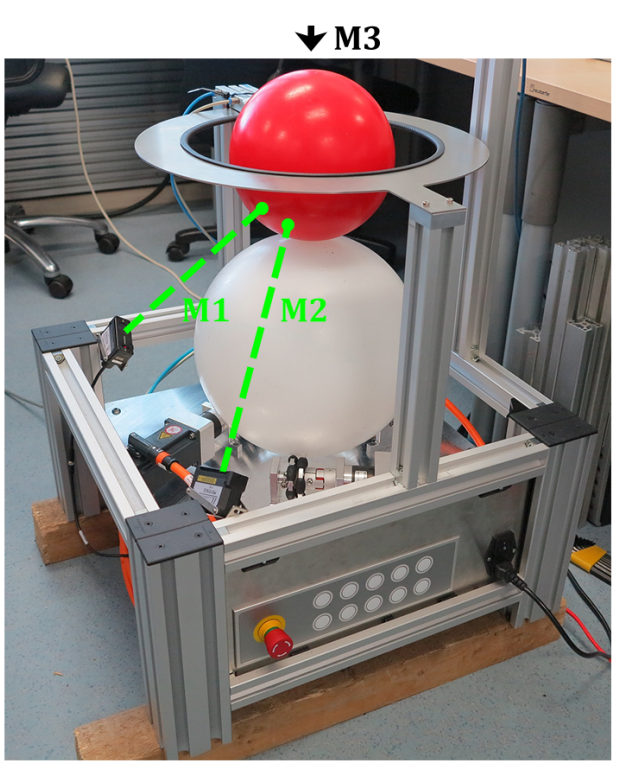

Fig. 7. Photograph of the experimental setup. The dashed green lines indicate the laser beam of the triangulation sensors and the black arrow indicates the camera view resolution of 0.5 Megapixels $(780 \times 580$ pixel $)$ at a frame rate of 67.5 frames per second $^{(16)}$. It is connected to the PLC and the images are directly transferred. Beckhoff TwinCAT vision module is used to calculate the position and the angles of the upper ball. As the lower ball is white and the upper ball red, it is the most intuitive approach to use just the red color channel of the RGB image to detect the center of mass of the ball and calculate the angles. This method is quite robust against wrong color pixels as they don't really influence the center of mass due to averaging. When the illumination is changed due to different light conditions also the thresholds for the red detection have to change multiple times a day. This leads to a weak contour detection and therefore to an unreliable measurement. The calculation duration for the selected image size of $580 \times 580$ pixel is about $650 \mu \mathrm{s}$.

To get a more reliable measurement the RGB image is transformed to the Hue Saturation Value (HSV) color space with the brightness as parameter ${ }^{(17)}$. The transformation of the image to the HSV space has a duration of about $2 \mathrm{~ms}$. To decrease this time not the full image is transformed, instead two stripes at the upper rest position in orthogonal directions are taken from the image and transformed. After the transformation the color detection and the mass center detection are done in the HSV color space. The whole optimized procedure takes about $800 \mu \mathrm{s}$.

\section{Results}

In Fig. 8 the residual error of the stabilized system can be seen. The system can be stabilized with the PD and the nonlinear controller with a sample time of $10 \mathrm{~ms}$. It can be seen, that the RMS error of both control methods is nearly the same. To evaluate the performance of the vision feedback the stabilization of the upper ball is done with the nonlinear controller and the camera measurement, because in a further work the nonlinear control should be extended by a trajectory following. The result can be seen in Fig. 9. The nonlinear controller with camera feedback (blue) shows the same performance for a sampling time of $10 \mathrm{~ms}$ as the nonlinear controller with the laser distance sensors (LDS). The total sampling time of the camera position measurement is approx. $15.8 \mathrm{~ms}$ while the LDS needs approx. $3 \mathrm{~ms}$. The measurement task of the camera and the controller task are completely separated. So it can happen that the controller gets an update of the ball position between two iterations or not. Of course this affects the control performance, but the controller is still able to stabilize the upper ball with nearly the same performance as with the LDS measurement.

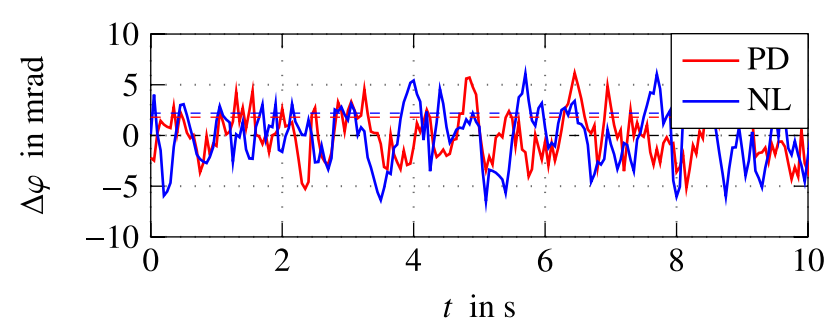

Fig. 8. Residual control error of the PD control $(1.80 \mathrm{mrad}$ RMS $)$ and the nonlinear control $(2.20 \mathrm{mrad}$ RMS) when using the laser distance sensors. RMS values are drawn dashed 


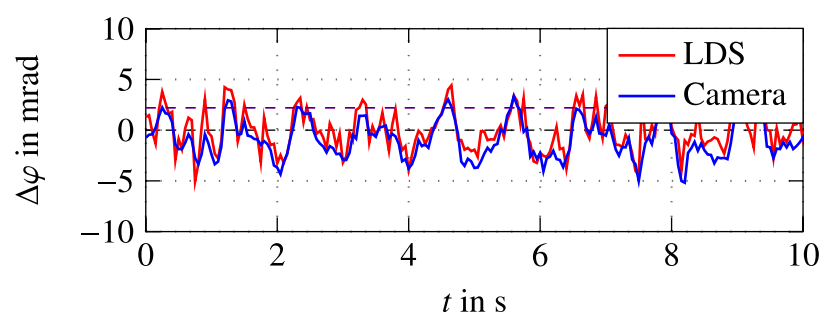

Fig. 9. Residual control error of the laser distance sensors (2.20 mrad RMS) and the camera (2.21 mrad RMS) using nonlinear control. RMS values are drawn dashed

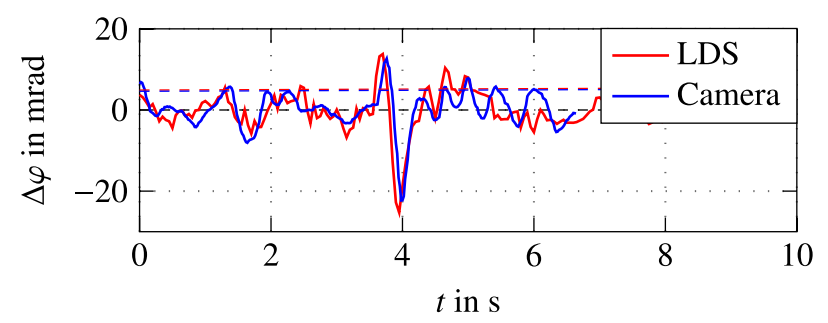

Fig. 10. Disturbance reaction by a pneumatic actuator at $3.6 \mathrm{~s}$ of the NL control using LDS (4.91 mrad RMS) and camera position measurement (4.61 mrad RMS). RMS values are drawn dashed

To show the disturbance rejection a pneumatic actuator is mounted on the system to get a reproducible disturbance for the upper ball position. The angular disturbance is about $13.8 \mathrm{mrad}$ at $3.6 \mathrm{~s}$. The reaction of the system on a disturbance can be seen in Fig. 10. The RMS values of the controller with the LDS measurement and the vision measurement are nearly equal and with both measurement methods the NL controller is able to keep the upper ball on top of the lower ball.

\section{Conclusion}

In this work a ball on ball setup is modeled, and linear and nonlinear controllers are designed and implemented. The measurement of the upper ball position is done with LDS and camera based. A state observer is introduced to determine the angular velocities of the upper ball. The experiments on the built setup show that it is possible to stabilize the ball on ball system with both feedback systems with a similar performance, and also a good disturbance rejection can be achieved. The complete control task and the image processing being performed on a PLC has been successfully demonstrated.

\section{Acknowledgment}

The support of Beckhoff Automation $\mathrm{GmbH}$ for providing the hardware and software, and Mr. Clemens Maier from Beckhoff for his assistance is gratefully acknowledged.

\section{References}

(1) A.J. Koivo and N. Houshangi: "Real-time vision feedback for servoing robotic manipulator with self-tuning controller", IEEE Transactions on Systems, Man, and Cybernetics, Vol.21, No.1, pp.134-142 (1991)

( 2 ) M. Bertozzi and A. Broggi: "Gold: A parallel real-time stereo vision system for generic obstacle and lane detection", IEEE Transactions on Image Processing, Vol.7, No.1, pp.62-81 (1998)

( 3 ) R. Gahleitner: "Ball on ball: Modeling and control of a novel experiment set-up", IFAC-PapersOnLine, Vol.48, pp.796-801 (2015)

( 4 ) S.-Y. Liu, Y. Rizal, and M.-T. Ho: "Stabilization of a ball and sphere system using feedback linearization and sliding mode control", in 8th Asian Control Conference (2011)

( 5 ) M.-T. Ho, Y. Rizal, and W.-S. Cheng: "Stabilization of a vision-based ballon-sphere system", in IEEE International Conference on Control Applications (2013)

( 6 ) H. Goldstein, J.C.P. Poole, and S.J.L. Safko: "KlassischeMechanik", WileyVCH (2012)

( 7 ) B. Siciliano, L. Sciavicco, L. Villani, and G. Oriolo: Robotics-Modeling, Planning and Control, Springer Verlag (2009)

( 8 ) R. Fischer: Elektrische Maschinen, Hanser Fachbuchverlag (2003)

( 9 ) H.K. Khalil: Nonlinear Systems (3rd edition), Pearson (2001)

(10) R. Munnig Schmidt, G. Schitter, and J. van Eijk: High Performance Mechatronics, Delft University Press (2011)

(11) G.F. Franklin, J.D. Powell, and M.L. Workman: Digital Control of Dynamic Systems, Addison Wesley Longman (1998)

(12) I.D. Landau and G. Zito: Digital Control Systems, Springer Verlag (2005)

(13) S. Fuchshumer, K. Schlacher, G. Grabmair, and K. Straka: "Flachheitsbasierte Folgeregelung des Labormodells Ball on the Wheel", e\&i, No.9, pp.301-306 (2004)

(14) R.E. Kalman: "A new approach to linear filtering and prediction problems", Transaction of the ASME, Journal of Basic Engineering, pp.35-45 (1960)

(15) F. Thomas and L. Ros: "Revisiting trilateration for robot localization", IEEE Transactions on Robotics, Vol.21, No.1, pp.93-101 (2005)

(16) GigE Vision Cameras Manta Series Technical Manual, V7.8.0 ed., AlliedVision, 01 (2017)

(17) A.R. Smith: "Color gamut transform pairs", ACM SIGGRAPH Computer Graphics, Vol.12, No.3, pp.12-19 (1978)

Matthias Bibl (Member) is research assistant in the Advanced

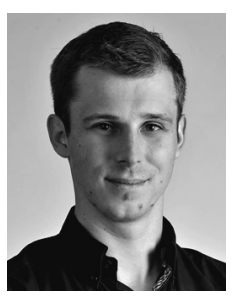
Mechatronic Systems group at the Automation and Control Institute (ACIN) of TU Wien. He received an MSc in Electrical Engineering from TU Wien, Austria (2014). His primary research interests are on automation systems, metrology systems and precision engineering.

Christoph Zech (Non-member) is developer at Beckhoff Automation

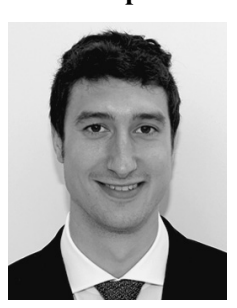
$\mathrm{GmbH} \& \mathrm{CoKG}$ in Kottingbrunn. He received an MSc in Electrical Engineering from TU Wien, Austria (2017). His primary research interests are on automation systems and robotic systems.

Georg Schitter (Non-member) is Professor for Advanced Mecha-

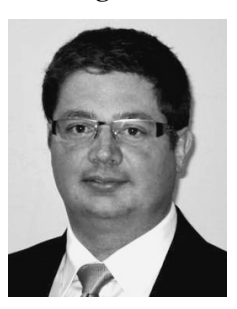
tronic Systems at the Automation and Control Institute (ACIN) of TU Wien. He received an MSc in Electrical Engineering from TU Graz, Austria (2000) and an MSc and PhD degree from ETH Zurich, Switzerland (2004). His primary research interests are on high-performance mechatronic systems, particularly for applications in the high-tech industry, scientific instrumentation, and mechatronic imaging systems, such as AFM, scanning laser and LIDAR systems, telescope systems, adaptive optics, and lithography systems for semiconductor industry. He received the IFAC Mechatronics best paper award (2008 to 2011) and 2013 IFAC Mechatronics Young Researcher Award, and served as an Associate Editor for IFAC Mechatronics, Control Engineering Practice, and for the IEEE-Transactions on Mechatronics. 